Article

\title{
An Optimal Energy-Saving Strategy for Home Energy Management Systems with Bounded Customer Rationality
}

\author{
Guoying Lin ${ }^{1}$, Yuyao Yang ${ }^{1}$, Feng Pan ${ }^{1}$, Sijian Zhang ${ }^{1}$, Fen Wang ${ }^{2}$ and Shuai Fan ${ }^{2, *}$ \\ 1 Metrology Center of Guangdong Power Grid Co., Ltd., Guangzhou 510080, China; \\ linguoying@gd.csg.cn (G.L.); yangyuyao@gd.csg.cn (Y.Y.); panfeng@gd.csg.cn (F.P.); \\ zhangsijian@gd.csg.cn (S.Z.) \\ 2 Key Laboratory of Control of Power Transmission and Conversion, Ministry of Education, Department of \\ Electrical Engineering, Shanghai Jiao-Tong University, Shanghai 200240, China; 5137509015@sjtu.edu.cn \\ * Correspondence: fanshuai@sjtu.edu.cn
}

Received: 6 March 2019; Accepted: 29 March 2019; Published: 2 April 2019

\begin{abstract}
With the development of techniques, such as the Internet of Things (IoT) and edge computing, home energy management systems (HEMS) have been widely implemented to improve the electric energy efficiency of customers. In order to automatically optimize electric appliances' operation schedules, this paper considers how to quantitatively evaluate a customer's comfort satisfaction in energy-saving programs, and how to formulate the optimal energy-saving model based on this satisfaction evaluation. First, the paper categorizes the utility functions of current electric appliances into two types; time-sensitive utilities and temperature-sensitive utilities, which cover nearly all kinds of electric appliances in HEMS. Furthermore, considering the bounded rationality of customers, a novel concept called the energy-saving cost is defined by incorporating prospect theory in behavioral economics into general utility functions. The proposed energy-saving cost depicts the comfort loss risk for customers when their HEMS schedules the operation status of appliances, which is able to be set by residents as a coefficient in the automatic energy-saving program. An optimization model is formulated based on minimizing energy consumption. Because the energy-saving cost has already been evaluated in the context of the satisfaction of customers, the formulation of the optimization program is very simple and has high computational efficiency. The case study included in this paper is first performed on a general simulation system. Then, a case study is set up based on real field tests from a pilot project in Guangdong province, China, in which air-conditioners, lighting, and some other popular electric appliances were included. The total energy-saving rate reached $65.5 \%$ after the proposed energy-saving program was deployed in our project. The benchmark test shows our optimal strategy is able to considerably save electrical energy for residents while ensuring customers' comfort satisfaction is maintained.
\end{abstract}

Keywords: energy saving; electric appliance utility function; prospect theory; energy-saving cost

\section{Introduction}

With the development of the economy, the share of electric power consumption of residential customers has dramatically increased, especially in developed regions [1]. In order to improve customers' energy efficiency, home energy management systems (HEMS) have been widely studied [2-4]. Generally, the HEMS can schedule electric appliances' operation status (ON/OFF status or comfortable inside temperature) according to the setpoints of customers [5]. However, these methods depend on the customers' setpoints, and cannot automatically and intelligently help people make optimal decisions. In recent studies, some researchers have proposed optimal energy 
strategies, which schedule the electric appliances to minimize energy cost while maximizing customer comfort satisfaction [6,7]. In order to realize this objective, two key problems should be tackled. The first is how to quantitatively evaluate customers' satisfaction, considering both energy-saving performance and the risk of comfort loss. On the other hand, we need to study how to optimally schedule the electric appliances' operation status based on the satisfaction evaluation indexes [8,9].

As for the first problem, many recent researchers have evaluated customer comfort from the perspective of indoor environmental quality (IEQ) [10]. For instance, [11,12] solved the energy-saving problem in buildings through structural design and feedback techniques, respectively. Although theses IEQ-based methods are able to improve comfort and energy saving, they focus more on inhabitants' behaviors and habits, and the planning and optimization before the fact. In a real-time energy-saving optimal control field, utility functions have more usually been used in recent research to evaluate customers' income when electricity is consumed. $[13,14]$ used a utility function that has a positive marginal return and a diminishing marginal rate of return from an economic viewpoint. In general, a logarithmic function, a diamond growth model, and a quadratic function are all suitable choices [15]. However, customers are assumed to be entirely rational in conventional utility functions. Utility functions are a concept from economic theories, while electricity is not wholly the same as general goods. Besides utility functions, some physical indexes are also adopted to evaluate the satisfaction of customers in electricity consumption. For instance, [16] used the real temperature to assess the performance of a chiller, while the state of charge was used to control the energy storage systems [17]. Physical indexes can only be used for one or two individual devices, and are not universal in residential energy-saving because there are various types of appliances in HEMS. To tackle this problem, conventional utility functions are improved by considering customers' bounded rationality, based on concepts from behavioral economics [18]. Accordingly, in our previous work, the utility function of electric appliances was combined with prospect theory for the sake of quantifying the customers' bounded rationality $[19,20]$. However, only several specific electric appliances were studied. In this paper, electric appliances are not categorized from a new perspective. We utilize the standard categories of time-sensitive and temperature-sensitive appliances, which cover nearly all the efficient electric appliances of residential customers. Based on our new clustering method, we extend the utility function into a new concept-energy-saving cost-while considering prospect theory to better depict the real behavior of customers. In conclusion, the proposed energy-saving cost is able to measure the satisfaction of a customer before and after optimization. Based on this measurement, we are able to formulate the optimization problem for energy saving, where the energy-saving is used as a constraint to make sure a customer's comfort will not be reduced.

Besides the satisfaction evaluation problem, how to optimize the appliances' commitments is the second issue in energy-saving programs. Recent researchers have usually modeled it as an optimization program from the day-ahead perspective. For example, [21,22] analyzed historical power consumption data, and formulated the energy-saving problem as a day-ahead offline optimization. Then, mixed-integer linear programming (MILP) can be derived from the original problem and computationally worked out. However, customers' satisfaction is not included in detail, and a day-ahead form is not robust to uncertainties. Additionally, machine learning techniques are adopted in some papers $[23,24]$. These studies are able to identify electricity scenarios using machine learning networks, while also determining the energy-saving strategy according to the relevancy between separate appliances and scenarios. In our previous work, Hidden Markov Model (HMM) methods were also implemented in the energy-saving project, but only in a small-scale pilot project [25]. It is worth noting that these machine learning-based methods are still not practical for many residents, due to the limited computational capability of HEMS. Thanks to the prospect theory-based utility function proposed in this paper, we tackled the energy-saving problem from the perspective of a real-time evaluation. Roughly speaking, we only need to maximize the utility function at each time slot, while minimizing the energy cost. This can be formulated as a straightforward mathematics problem, which ensures that the optimal strategy can be derived efficiently. 
The contributions of this paper can be summarized as follows.

(1) In order to define a utility function for all kinds of electric appliances for residents, this paper categorizes general appliances into two types: The time-sensitive type, and the temperature-sensitive type. Based on this clustering, we extend the utility function leveraged in $[13,14]$ into two types, covering more kinds of electric appliances in HEMS.

(2) Considering the bounded rationality of customers in an energy-saving program, prospect theory in behavioral economics is incorporated to extend the utility functions into a novel concept; the energy-saving cost. This depicts the real behavior of customers in energy-saving better than conventional utility functions.

(3) An energy-saving cost-based optimal appliance scheduling model is formulated. In our model, the energy-saving cost is used as a constraint, and HEMS only need to minimize the electric power consumption in acceptable regions. The method is computationally efficient, making it suitable for use with HEMS.

The remainder of this paper is organized as follows. Section 2 introduces the time-sensitive utility function and temperature-sensitive utility function, and the energy-saving cost is also defined. Section 3 proposes the optimal energy-saving model based on energy-saving cost. A case study is shown in Section 4, and the conclusions are outlined in Section 5.

\section{Electric Appliance Utility Function and Energy-Saving Cost}

\subsection{Introduction of Definitions}

Inspired by economics, the concept of utility is introduced to represent the relationship between appliance operation and user satisfaction. The electric appliance utility function is defined as customers' comfort satisfaction from the electric activity. The appliance utility is different from the utility concept in economics because electricity consumption, which is not a measurable, tangible good, currency or service, produces an influence on user satisfaction through the operation process of the electrical appliance. When users consume electricity, they do not consume the direct products of electricity, but rather indirect products into which electricity is converted through an appliance's operation, such as adjustment of the temperature of the air conditioner or the brightness of the electric light. Thus, different electrical appliances have different appliance utility functions. To cover all kinds of electric appliances for residents, appliance utility functions are defined by two categories; time-sensitive and temperature-sensitive.

\subsection{Time-Sensitive Utility Function}

The time-sensitive utility function quantifies customers' satisfaction with the actual appliance operation status of time-type appliances, such as electric lights. We define the operating status $s_{i}(t)$ of appliance $i$ at the moment $t$ as a binary variable, corresponding to two statuses: On and off. For the sake of simplicity, the time-sensitive utility function is expressed as time utility.

It is generally believed to be high energy efficiency for electrical appliances to operate at the time when the user needs them. However, for practical engineering implementation, it is difficult to decide whether the user needs to use the appliance at a particular time. Instead, the historical electricity consumption data can be mined to calculate the operating probabilities of an appliance, which is regarded as the probability that the user needs to use the appliance, marked as $P^{o n}$.

As a result, the time utility function is shown as Equation (1):

$$
U_{i}^{\text {time }}(t)=\left\{\begin{array}{ll}
\rho\left(P_{i, t}^{o n}(H)\right) & s_{i}(t)=1 \\
1-\rho\left(P_{i, t}^{o n}(H)\right) & s_{i}(t)=0
\end{array} .\right.
$$


In this equation, $U_{i}^{\text {time }}(t)$ represents the time utility value of appliance $i$ at the moment of $t$, and $H$ is the parameter that is set to infer $P_{i, t}^{o n}$, which is the operating probability of appliance $i$ at the moment of $t$. The function $\rho(x)$ is the system preference function, which is introduced to represent decision preferences given from a decision-making system, defined as Equation (2). It can be seen from Equation (1) that when the operating probability of an electrical appliance is more significant at a certain time, user satisfaction is higher.

$$
\rho(x)= \begin{cases}1 & x \in\left((2 \alpha)^{-\frac{1}{3}}+0.5,1\right] \\ \alpha(x-0.5)^{3}+0.5 & x \in\left[-(2 \alpha)^{-\frac{1}{3}}+0.5,(2 \alpha)^{-\frac{1}{3}}+0.5\right] . \\ 0 & x \in\left[0,-(2 \alpha)^{-\frac{1}{3}}+0.5\right)\end{cases}
$$

In Equation (2), $\alpha$ is the decision preference coefficient, and $\rho(x)$ is the system preference function of converting the objective probability into the preference probability, in which $x$ is the objective probability value. In general, things that have an extremely small probability are often regarded as impossible events. On the contrary, things that have a significant probability are seen as absolute events. In conformity with this, the output $\rho(x)$ in Equation (2) is the decision preference probability, which ignores impossible events and affirms very likely events. The preference extent depends on $\alpha$, which ranges from 4 to infinity. A bigger $\alpha$ corresponds to a larger preference extent.

\subsection{Temperature Utility Function}

The temperature-sensitive utility function is for appliances, such as air-conditioners and water heating dispensers, which impact residents through changing the temperature. It quantifies the relationship between user satisfaction and the actual temperature according to the relationship between the expected temperature and the actual temperature, shown as Equation (3).

$$
U_{i}^{t e m p}\left(T_{t}\right)= \begin{cases}e^{-\varepsilon\left(T_{t}-T_{l}\right)^{2}} & T_{t}<T_{l} \\ 1 & T_{t} \in\left[T_{l}, T_{h}\right] \\ e^{-\varepsilon\left(T_{t}-T_{h}\right)^{2}} & T_{t}>T_{h}\end{cases}
$$

In the equation, $U_{i}^{\text {temp }}\left(T_{t}\right)$ represents the temperature utility value of appliance $i$ at the moment of $t, T_{t}$ is the actual temperature at the time of $t, \varepsilon$ is the temperature utility falling coefficient, and $\left[T_{l}, T_{h}\right]$ is the prospective temperature threshold value. The utility value equals 1 when $T_{t}$ varies between $T_{t}$ and $T_{h}$. When $T_{t}<T_{l}$ or $T_{t}>T_{h}$, the utility value decreases gradually from 1 at a certain falling speed. The falling speed depends on $\varepsilon$, which changes from 0 to 1 . The smaller the $\varepsilon$, the faster the descent speed.

\subsection{Energy-Saving Costs}

Under the condition of guaranteeing user satisfaction as much as possible, energy-saving optimization for electrical appliances refers to adjusting the operation status to the most energy-saving status. In order to quantitively evaluate the comfort loss risk for the customers in the energy-saving program, this paper proposes a new concept: The energy-saving cost.

The energy-saving cost is defined as the difference of the utility function value before and after energy-saving optimization, shown as Equation (4).

$$
\hat{C}_{i}(t)=U_{i}(t)-\hat{U}_{i}(t) \text {. }
$$

In this equation, $\hat{C}_{i}(t)$ denotes the energy-saving costs of appliance $i$, accepting a certain optimization strategy at the time slot $t . U_{i}(t)$ is the appliance utility value before executing the procedure, and $\hat{U}_{i}(t)$ is appliance utility value after implementing the strategy. If energy-saving costs are positive, it indicates that the energy-saving strategy will weaken user satisfaction, 
but if energy-saving costs are negative, it represents that user satisfaction will not be damaged by optimization.

Since the calculation of time utility is based on probability, misoperation cannot be avoided entirely. Once misoperation occurs, user satisfaction will sharply drop to near zero. Thus, for time-type appliances, a further definition of energy-saving costs is proposed to avoid misoperation for those operations that may lower utility value, shown as Equation (5).

$$
\hat{C}_{i}(t)= \begin{cases}U_{i}(t)-0 & \gamma U_{i}(t) \geq \hat{U}_{i}(t) \\ U_{i}(t)-\hat{U}_{i}(t) & \gamma U_{i}(t)<\hat{U}_{i}(t)\end{cases}
$$

In this equation, $\gamma$ is the energy-saving risk control factor, which can affect the misoperation risk of the energy-saving strategy. If $\gamma$ equals 1 , users tend to refuse those operations which may lower appliance utility. A smaller $\gamma$ means that users are more willing to take the risk of misoperation. On the contrary, the larger $\gamma$ is, the less willing they are to accept misoperation.

Then, considering the bounded customer rationality, prospect theory in behavioral economics [18] is introduced into the energy-saving costs to evaluate the relationship between energy-saving and user satisfaction. The energy-saving costs evaluation method is shown as Equation (6), which is a mapping of the energy-saving costs to the users' subjective feeling for energy-saving costs.

$$
C_{i}(t)=\left\{\begin{array}{ll}
\eta \cdot \hat{C}_{i}(t)^{\theta} & \hat{C}_{i}(t) \geq 0 \\
-\left(-\hat{C}_{i}(t)\right)^{v} & \hat{C}_{i}(t)<0
\end{array} .\right.
$$

In this equation, $\eta$ is the sensitive coefficient for energy-saving costs, $\theta$ is the risk aversion coefficient for utility damage, which belongs to $(0,1]$, and $v$ is the risk preference coefficient for utility gain, which has the same range. The larger $\eta$ is, the less willing users are to accept energy-saving strategies which will influence user satisfaction.

Furthermore, it is worth noting that energy-saving costs include both instantaneous energy-saving costs and accumulative energy-saving costs. In the time dimension, changes of appliance operation status will influence the appliance utility instantaneously, so that instant energy-saving costs can accurately characterize the gain or damage of user satisfaction. This is defined as Equation (6). On the other hand, in the temperature dimension, changes in appliance operation status cannot affect the appliance utility instantaneously when the control instruction is issued. For instance, when changing the air conditioner operation status (such as by turning on, turning off, or adjusting the set temperature), the environmental temperature does not change instantaneously, but changes gradually. Not only the trend, but also the magnitude of the change, depends on the specific setting of the new mode. In this situation, accumulative energy-saving costs for a continuous period characterizes the impact of the energy-saving strategy on user satisfaction. Defined as Equation (7), the accumulative energy-saving cost is calculated for a continuous period of time.

$$
S C_{i}(t)=\int_{t_{s}}^{t_{e}} C_{i}(t) d t .
$$

\section{Energy-Saving Optimization Model}

\subsection{Automatic Recognition of Appliance Status}

Before optimization, it is necessary to achieve real-time recognition of the operating state of the electrical appliances. The recognition method consists of two parts: The offline dictionary formulation, and online recognition. The automatic recognition algorithm was revealed in our previous work [26], and it is not the contribution of this paper, so here we introduce it in a simple form. First, based on HEMS [8], which include smart plugs and gateways, various experiments can be used to collect and store different kinds of electric appliances' operation data. Then, we can use the K-Means algorithm to 
cluster the power data to several centers [27], in which each center corresponds to a typical operating state. It is worth noting that the number of centers depends on some previous knowledge, but is not like the method in [27]. For example, it is easy to know that a water dispenser has two typical operation states, including heating and insulation. After the offline experiments, a dictionary is obtained which is a set of typical operation states, and can be used for online recognition. In the real-time operation, the new data collected in real time is compared with the typical operation states in the dictionary. In this way, we can obtain real-time operation states of an appliance at a given time slot.

An example is given as follows. We used the K-Means algorithm to cluster several days of real power data of an electric light and water dispenser (time-type appliances), and an air conditioning unit (temperature-type appliance). The test data comes from the filed data in a pilot project in Guangzhou Province, China. The respective clustering centers were obtained for each appliance, which corresponded to the typical operation status of the appliances. Table 1 shows our clustering results. For instance, it is not difficult to know that a water dispenser has two typical operating statuses: Insulation and heating. Accordingly, two cluster centers are obtained, including $0.469 \mathrm{~W}$ and $426.26 \mathrm{~W}$. As a result, we can assert that if the collecting power value in real time is near to $0.469 \mathrm{~W}$, the operating status should be insulation, while if the collecting power value in real time is near to $426.26 \mathrm{~W}$, the operating status should be heating. We also undertook real-time recognition tests, as shown in Figure 1. In the test, the real-time monitored data from smart plugs were compared with the clustering results in Table 1 to obtain real-time operation status. Figure 1a shows the operation status recognition result of the air conditioning unit, and Figure $1 \mathrm{~b}$ displays the operation status recognition result of the water dispenser. For instance, the blue dots in Figure $1 \mathrm{~b}$ are thought to occur during heating status, because the values of these blue dots are near to the reference value of the heating status of a water dispenser in Table 1, of $426.26 \mathrm{~W}$.

Table 1. Reference values of operating status and characterized power.

\begin{tabular}{ccc}
\hline Appliances & Operating Status & Clustering Center/W \\
\hline Light & On & 58.28 \\
\hline \multirow{2}{*}{ Water Dispenser } & Insulation & 0.46928 \\
& Heating & 426.26 \\
\hline \multirow{3}{*}{ Air Conditioning } & Cooling & 1139.77 \\
& Heating & 1407.65 \\
& Ventilation & 56.25 \\
& Standby & 20.56 \\
\hline
\end{tabular}

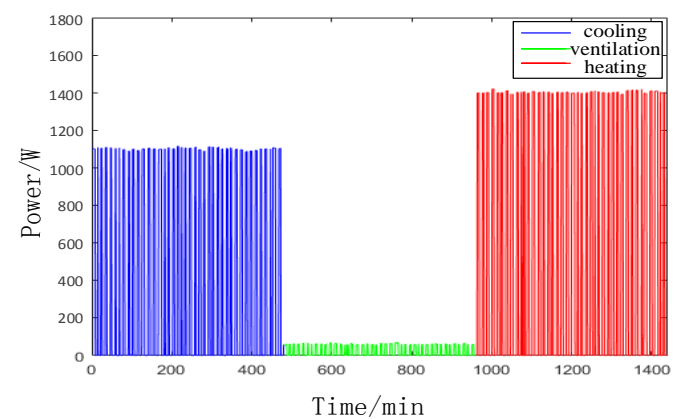

(a)

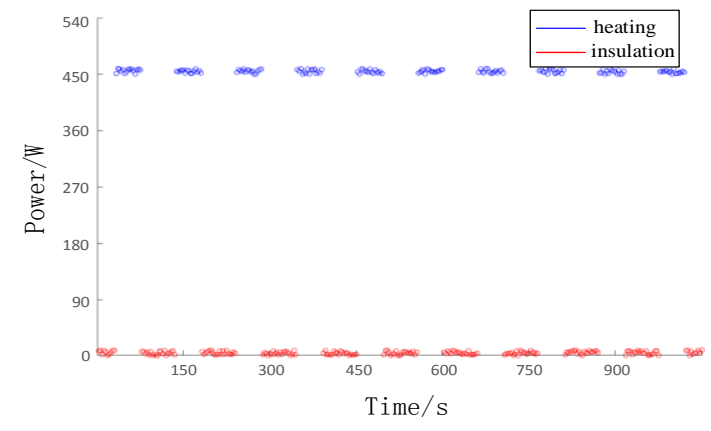

(b)

Figure 1. The operating status recognition results. (a) The operation status recognition of the air conditioning unit. (b) The operation status recognition of the water dispenser.

\subsection{Power Demand Estimation}

After status recognition, it is also necessary to estimate the power demand, which includes the time demand of time-type appliances and the temperature demand of temperature-type appliances. 
For the time demand of the time-type appliances, the power demand can be excavated by statistical analysis of the historical operation of the electric appliance, or by using the environmental sensors to monitor user activities, as shown in Equation (8), where $P_{i}^{o n}(t)$ represents the operating probability of appliance $i$ at the moment of $t, n_{\text {total }}$ denotes the total number of tests, and $n_{0}$ denotes the number of electricity-consumption occurrences. For the temperature demand of the temperature-type appliances, real-time monitoring can be performed through the temperature sensor.

$$
P_{i}^{o n}(t)=\frac{n_{0}}{n_{\text {total }}}
$$

After clarifying the power demand, the time/temperature utility is evaluated in conjunction with the identified operating status, along with the corresponding utility functions which have been previously defined.

\subsection{Energy-Saving Optimization Algorithm Flow}

Based on a clear evaluation method of energy-saving costs proposed in Section 2, an energy-saving optimization model for residential appliance management is formulated. We set energy-saving costs as a constraint, as shown in Equation (9), where $\xi$ is the risk coefficient in the energy-saving optimization strategy. When the probability of misoperation is smaller, the risk of energy saving is less. As for the objective function, we hope to maximize energy efficiency, or in other words, minimize the electricity consumption, as shown in Equation (10), where $E_{i}[0, t]$ is the electricity consumption and $p_{i}(t)$ is the power of appliance $i$ throughout $[0, t]$. This represents the instantaneous energy-saving model when $t_{a}=0$ in the time dimension, and represents the accumulative energy-saving model in the temperature dimension when $t_{a}>0$.

$$
\begin{gathered}
S C_{i}\left[t_{0}, t_{0}+t_{a}\right] \leq \xi \\
\min E_{i}\left[t_{0}, t_{0}+t_{a}\right]=\int_{t_{0}}^{t_{0}+t_{a}} p_{i}(t) d t .
\end{gathered}
$$

In particular, appliances of the same kind and of the same impact on electric effect can be combined for optimization, such as several air conditioners in the same area, when the total electricity consumption is set as the optimization target. Therefore, marking a set of the appliances which have the same impact on the electric effect in the region $r$ as $I_{r}^{n}$, and marking the electricity consumption within the period $[0, t]$ as $\min E_{I_{r}^{n}}[0, t]$, the target function is:

$$
\min E_{I_{r}^{n}}\left[t_{0}, t_{0}+t_{a}\right]=\int_{t_{0}}^{t_{0}+t_{a}}\left(\sum_{i \in I_{r}^{n}} p_{i}(t)\right) d t .
$$

Based on the model formulated above, the energy-saving optimization algorithm flow is designed in the time and temperature dimensions respectively, which are introduced in detail in the next subsections.

\subsubsection{Time Dimension}

Assuming time-type appliances only have two operating statuses (on/off), the key to energy-saving optimization for general time-type appliances (such as lights, televisions, and so on) in the time dimension is to decide whether an appliance should be on or off at a certain moment.

The time-type appliance optimization algorithm uses the real-time energy-saving costs as the evaluation basis, and the whole process is shown in Figure 2. 


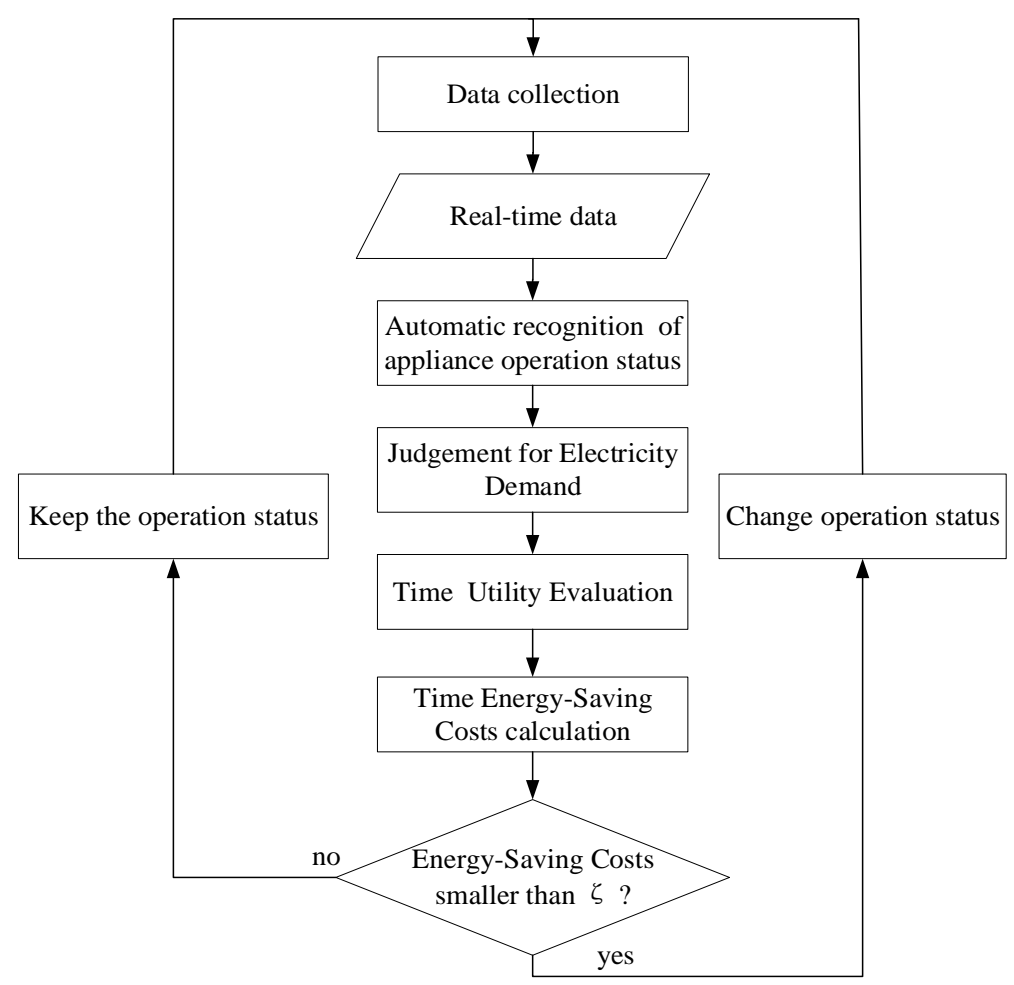

Figure 2. Energy saving optimization flow for time-sensitive appliances.

The above process can be divided into the following steps:

(1) Recognize the operating status of the electrical appliance using the automatic recognition method of the appliance operation status with real-time collected operating power data;

(2) Select the power demand mining method which applies to the appliance according to the characteristics of electrical appliances. Then, the current demand for power consumption is discriminated;

(3) Evaluate the real-time time utility according to the electrical appliance operation status and the power demand;

(4) Calculate the energy-saving costs if the electrical operation mode is changed (previously turned on, assumed to be off; or closed initially, and assumed to be on);

(5) Determine the relationship between the energy-saving costs and the energy-saving threshold $\xi$. If the energy-saving cost value is lower than $\xi$, the optimization strategy is to change the electrical operation mode; if it is higher than $\xi$, the optimization strategy is to keep the original operating mode unchanged.

\subsubsection{Temperature Dimension}

For temperature-type appliances like air conditioners, the key to optimization is to predict the environment changes of air conditioners in a future period. In the case of keeping the operating status unchanged in the coming period and adopting a certain control command to change the operating status, respectively, the indoor temperature is predicted with the first-order indoor temperature model proposed in [11], as shown in Equation (12).

$$
x^{\text {in }}[t+1]=x^{\text {in }}[t]+a\left(x^{\text {out }}[t]-x^{\text {in }}[t]\right)+g \cdot p^{H V A C}[t]+c .
$$

In this equation, $a$ is the sensitivity coefficient of the indoor and outdoor temperature difference, $g$ is the appliance's operating contribution coefficient, and $c$ is the constant parameter. The temperature 
utility value is pre-evaluated in combination with the corresponding operation status and the predicted indoor temperature value.

The process of energy-saving optimization in the temperature dimension is shown in Figure 3.

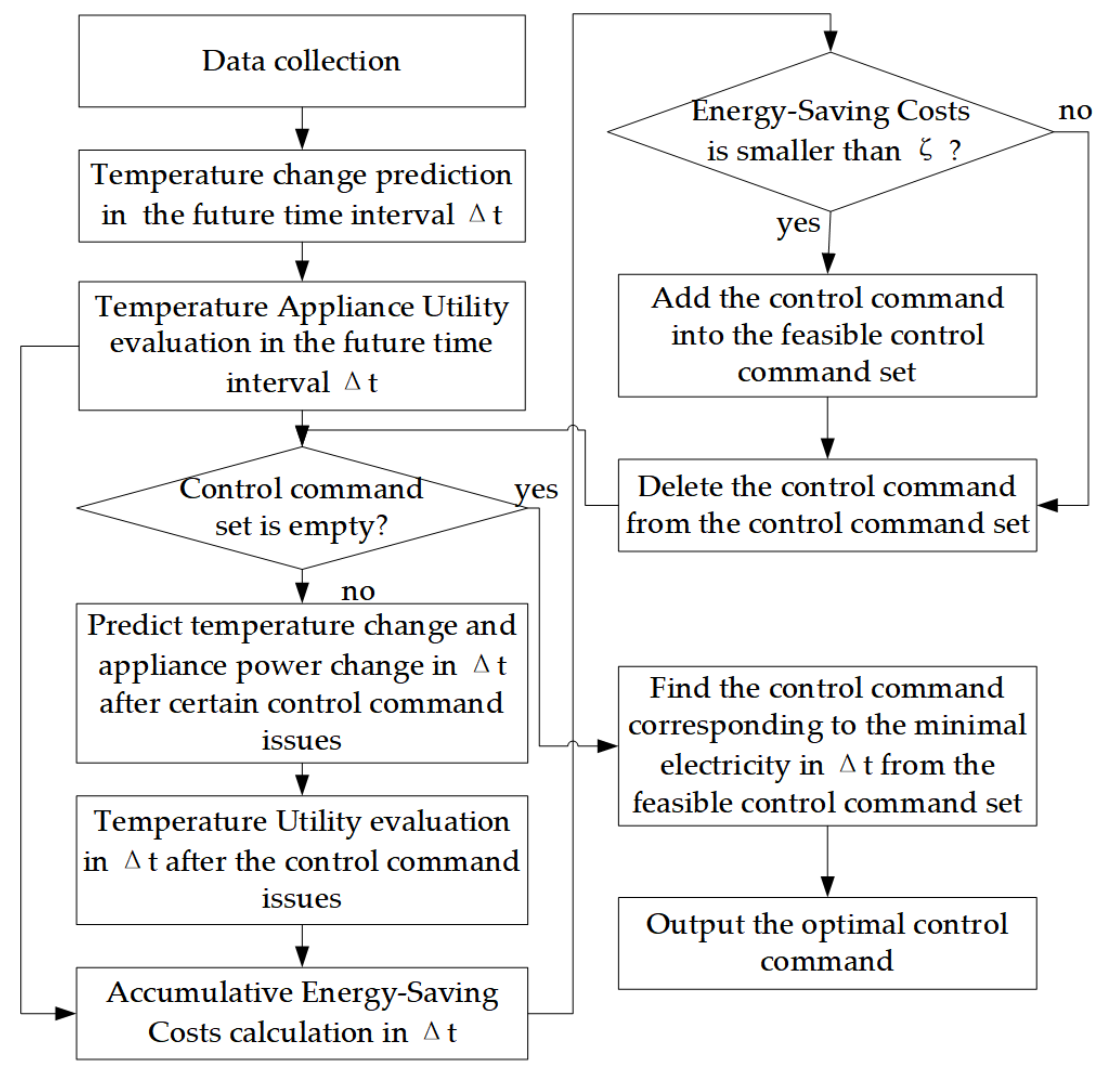

Figure 3. Energy saving optimization flow in the temperature dimension.

The above process can be divided into the following steps:

(1) Predict the indoor temperature change under the condition that the operating mode remains unchanged in the future period of $\Delta t$;

(2) Pre-evaluate the temperature utility during the future time period of $\Delta t$ in combination with the operating status and the indoor temperature prediction curve;

(3) Mark the set of all the currently operational control commands as the control command set, and determine whether the control command set is empty. If it is empty, go to (7); if not, go to (4);

(4) Select one of the operable control commands to predict the operating status, operating power, and indoor temperature change of the future period of $\Delta t$ after the control command is issued;

(5) Pre-evaluate the temperature utility by combining the new operation status and indoor temperature prediction;

(6) Combine the temperature utility estimation result before and after taking the control command, calculate the energy-saving costs brought by the control command, and judge whether it is less than the setting energy-saving temperature utility cost threshold $\xi$. If it is less than $\xi$, the control command is put into the available control command set and would be deleted from the control command set; if it is higher than $\xi$, delete the control command from the control command set. Then, return to step (4);

(7) Traverse all control commands of the available control command set, perform an integral calculation on the power curve predicted in (4), and find the control command with the smallest total power consumption, which is the optimal energy-saving control strategy. 
In particular, the cluster optimization of multiple air conditioners in the same area can be abstracted into the optimization problem of an air conditioner with multiple-gear operating power. In addition to turning on, turning off, and adjusting the set temperature, the control command of the air conditioner includes selecting a power gear position, so the operational control command set is a combination command for setting the temperature and the power gear position. After that, the optimization algorithm flow is consistent with a single air conditioner.

Combined with the operation principle of the fixed-frequency air conditioner and Formula (12), the power curve of the air conditioner and the indoor temperature in future periods are predicted, as shown in Figure 4.

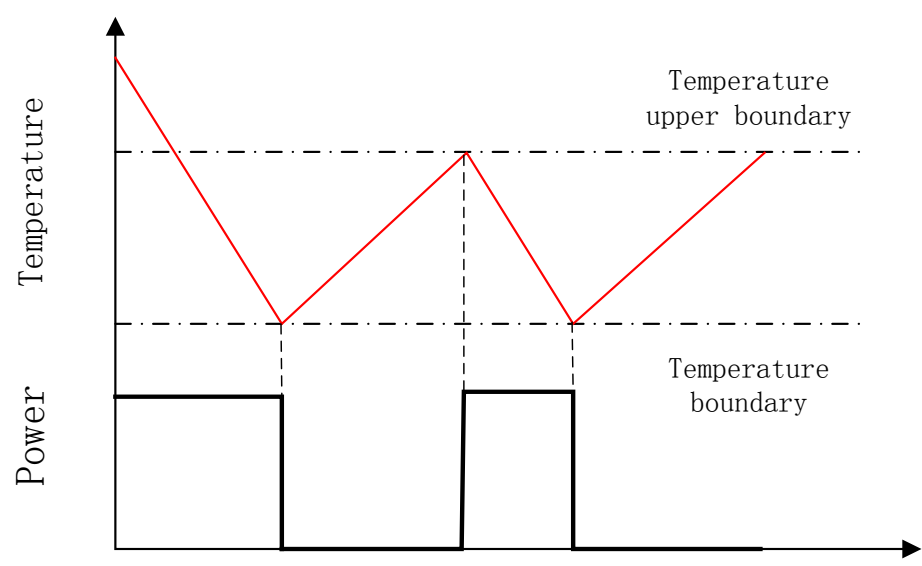

Figure 4. Running a dynamic process diagram for a fixed-frequency air conditioner.

\section{Case Study}

\subsection{The Basic Test of the Proposed Algorithm}

First, we undertook some simulations to test our algorithm. The corresponding parameters come from $[5,20]$, and are set as follows:

$$
\left\{\begin{array}{l}
{\left[T_{l}, T_{h}\right]=\left[23^{\circ} \mathrm{C}, 25^{\circ} \mathrm{C}\right]} \\
\varepsilon=1 / 64 \\
\alpha=16 \\
\gamma=\eta=1 \\
\theta=0.68 \\
v=0.88 \\
\xi=-0.1
\end{array}\right.
$$

As mentioned above, before the optimization, it is necessary to find the operating status of the electrical appliances and the implicit user power demand. The process of automatic recognition of the operating status is elaborated, so here the mining process for power demand is expounded. The power demand for lights and water dispensers in the time dimension is projected based on field research on the law of electrical appliance use, and historical electricity behavior statistics. For air conditioning, with the help of a temperature sensor, the power demand mining process in the temperature dimension is completed.

For example, for lights, infrared sensors are used to detect whether there is user activity in real time; that is, real-time monitoring of the time demand. For water dispensers, the time demand is obtained by performing a longitudinal analysis of the drinking behavior at each time section. The operation and the corresponding utility value of some appliances in a certain office are shown in Figure 5. In Figure 5a-c the operation of a certain light can be seen; Figure $5 d-f$ show the operation of a certain water dispenser; Figure $5 \mathrm{~g}-\mathrm{i}$ show the operation of a certain single air conditioner, where the 
satisfactory temperature range of the temperature utility function is set as $\left[T_{l}, T_{h}\right]=[24,26]$, and the corresponding temperature utility evaluation result is shown in Figure $5 f$.

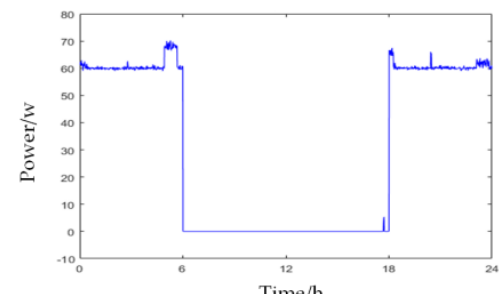

(a)

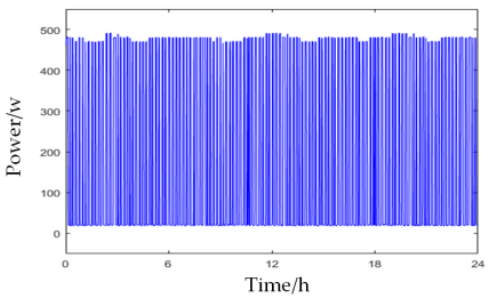

(d)

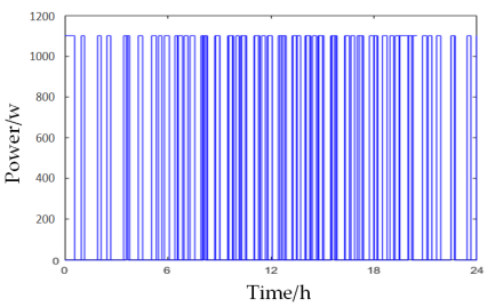

(g)

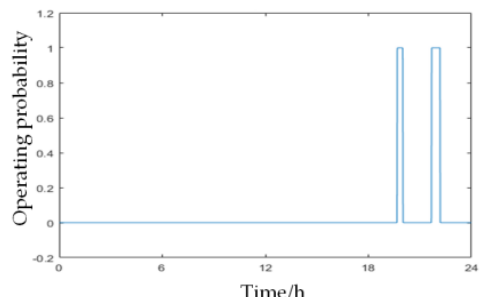

(b)

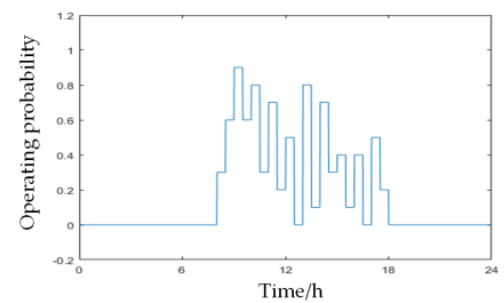

(e)

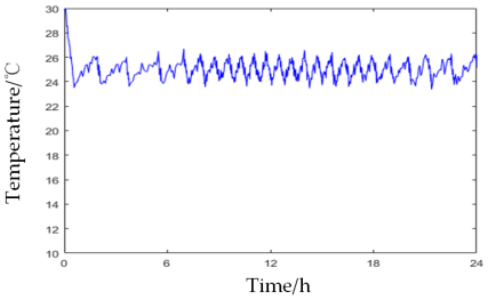

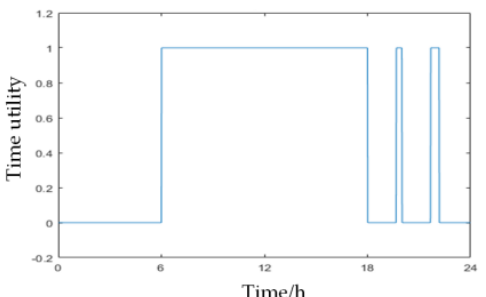

(c)

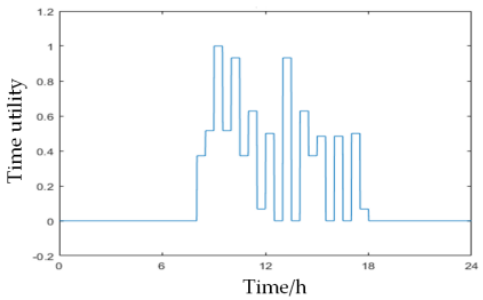

(f)

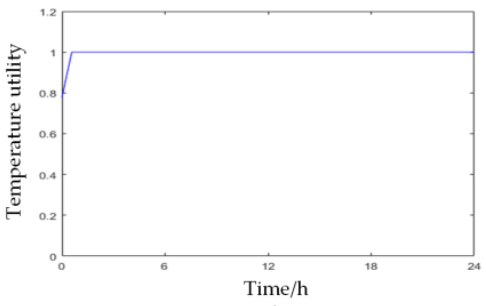

(i)

Figure 5. Utility evaluation of the test electrical appliances. (a) Light-power. (b) Time demand of lighting. (c) Time utility for lighting. (d) Water dispenser power. (e) Time demand of a water dispenser. (f) Time utility of a water dispenser. (g) Air conditioning power. (h) Indoor temperature. (i) Temperature utility of air conditioning.

\subsubsection{Optimization for Time-Sensitive Appliances}

As mentioned above, lights and water dispensers are two kinds of typical time-sensitive electric appliances. In this section, we chose them to test our energy-saving strategy for time-sensitive appliances. As shown in Figure 6, the running time of appliances greatly reduced, and the energy saving benefits were considerable. It is worth noting that the comfort loss risk for customers can be ensured in the optimization, due to the constraints of Equation (9). For instance, as shown in Figure 6c, the real running hours of the water dispenser (red solid line) reduced sharply after optimization, compared with the original operating hours (blue solid line). However, the value of the utility increased, as shown in Figure 6d. The reason for this is that the operating probability is zero from 0:00 to 8:00 and 18:00 to 24:00, as shown in Figure 5e, which means there was no drinking necessary. As a result, optimizing the operation status in these time slots from heating to insulation will increase the utility value and save more energy, while ensuring customer comfort. 


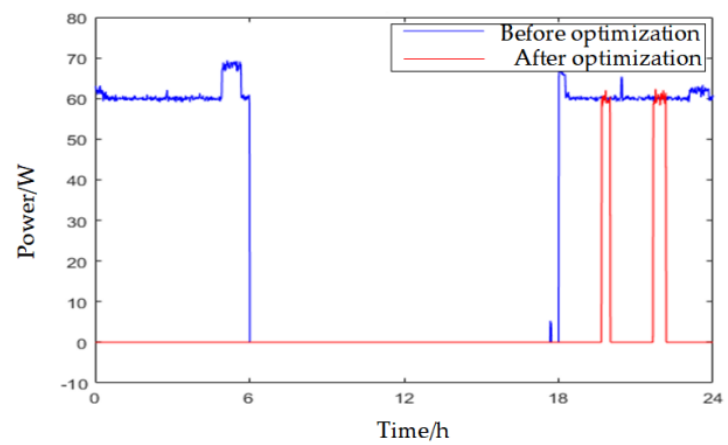

(a)

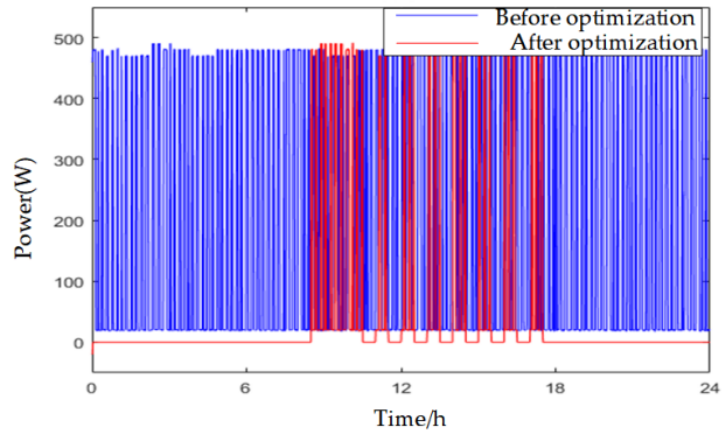

(c)

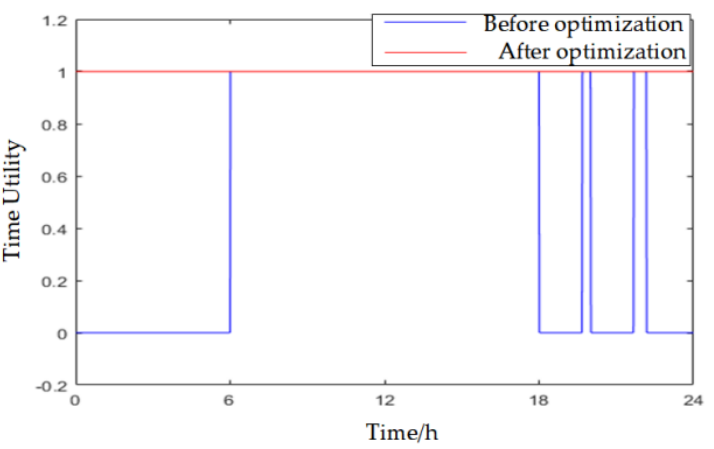

(b)

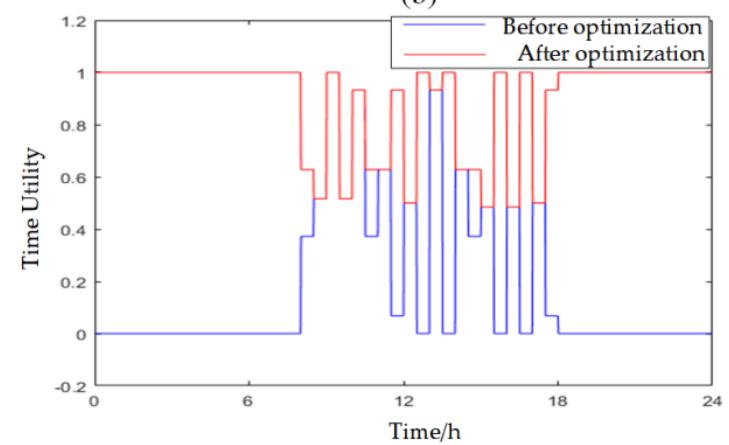

(d)

Figure 6. Energy saving optimization for lights and water dispensers. (a) Lighting power. (b) Lighting time utility. (c) Water dispenser power. (d) Water dispenser time utility.

\subsubsection{The Optimization Simulation for an Air Conditioner}

For a single air conditioner and air conditioner group, we undertook an optimization simulation with half an hour as the optimal period from the known outdoor temperature curve $\left(T_{\text {out }}=\left[26^{\circ} \mathrm{C}, 35^{\circ} \mathrm{C}\right]\right)$ on a certain summer day, and the result is shown in Figure 7 . The energy-saving data in the above air conditioner's simulation is shown in Table 2. It can be seen that a single air-conditioner needs to operate continuously when the outside temperature is too high, and the energy-saving potential is limited. However, for the air conditioning unit, when the total running time is shortened by adjusting the number of opening appliances and setting the proper temperature, the energy-saving effect is significant.

Table 2. The results of the energy saving simulation for an air-conditioner.

\begin{tabular}{ccccc}
\hline Appliances & $\begin{array}{c}\text { Before } \\
\text { Optimization/kWh }\end{array}$ & $\begin{array}{c}\text { After Optimization } \\
/ \mathbf{k W h}\end{array}$ & $\begin{array}{c}\text { The amount of Energy } \\
\text { Saving/kWh }\end{array}$ & $\begin{array}{c}\text { Energy Saving } \\
\text { Rate }\end{array}$ \\
\hline $\begin{array}{c}\text { Single air conditioner } \\
\begin{array}{c}\text { Air conditioner group } \\
\text { (four devices) }\end{array}\end{array}$ & 15.48 & 14.75 & 0.73 & $4.7 \%$ \\
\hline
\end{tabular}




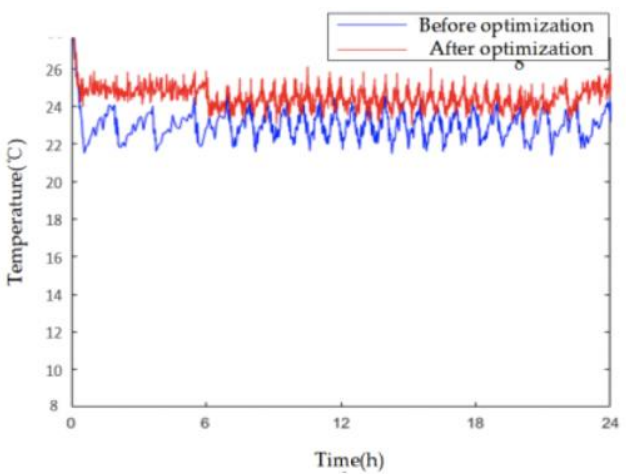

(a)

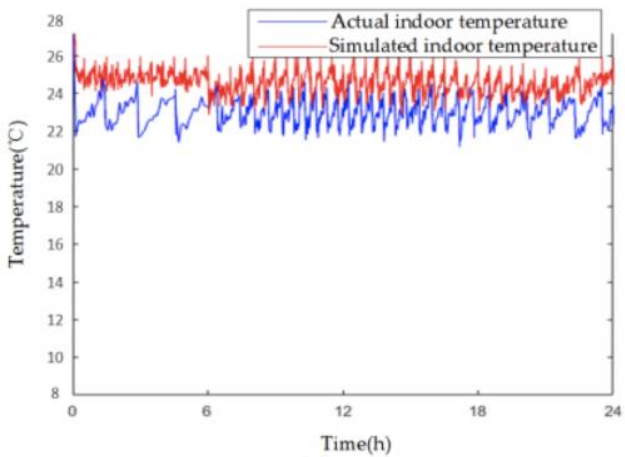

(c)

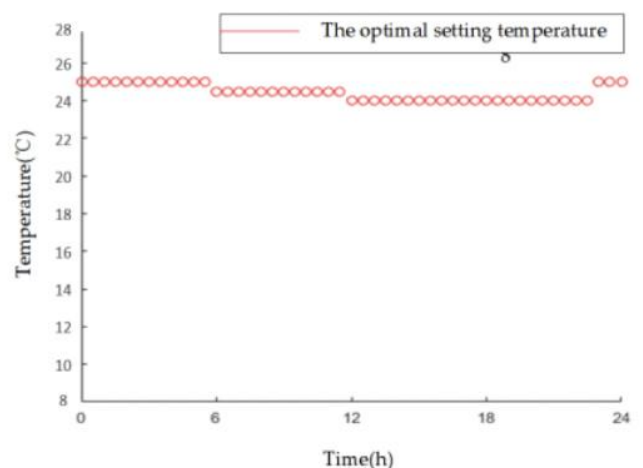

(b)

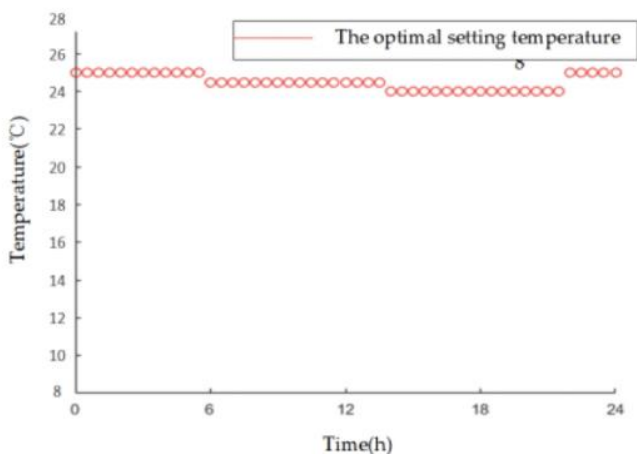

(d)

Figure 7. Energy saving optimization for air conditioning. (a) Indoor temperature of a certain office. (b) The optimal set temperature of the office. (c) Indoor temperature in a hyperbaric chamber. (d) The optimal set temperature of the hyperbaric chamber.

\subsection{The Energy Saving Effect Test Based on Field Data}

To obtain the actual value of the energy-saving effect, we performed our optimization strategies in a pilot project in Guangdong province. The project is located in Guangzhou, Guangdong province, China, and the electrical appliances are listed in Table 3.

Table 3. Electrical appliances in the pilot project.

\begin{tabular}{ccc}
\hline Type of Area & Appliances & Number \\
\hline Hyperbaric chamber/Central control room & Air conditioner & 6 \\
Office & Air conditioner & 3 \\
Office & Water dispenser & 3 \\
Outdoor area & Lighting & 5 \\
Indoor area & Lighting & 5 \\
\hline
\end{tabular}

To realize a real-time collection of power consumption data and remote control, smart plugs were installed for the time-type equipment (water dispensers, lighting equipment) in the pilot project, and smart plugs and intelligent infrared controllers were installed for the temperature-type equipment (air conditioners). Additionally, the temperature sensors were set to monitor the indoor and outdoor temperature in real time. Acoustic sensors were installed to monitor lighting requirements in real time.

Then, the above-mentioned energy-saving optimization algorithms were performed, and the power consumption for one week of the station was calculated before and after the implementation of the automatic energy-saving optimization strategy. Based on this, the energy-saving related indicators were computed. The results are shown in Table 4. 
Table 4. Energy saving statistics.

\begin{tabular}{cccccc}
\hline Appliances & $\begin{array}{c}\text { Before } \\
\text { Optimization/kWh }\end{array}$ & $\begin{array}{c}\text { After } \\
\text { Optimization/kWh }\end{array}$ & $\begin{array}{c}\text { Total Energy } \\
\text { Savings/kWh }\end{array}$ & $\begin{array}{c}\text { Energy-Saving } \\
\text { Rate }\end{array}$ & $\begin{array}{c}\text { Ratio of } \\
\text { Energy-Saving }\end{array}$ \\
\hline $\begin{array}{c}\text { Air conditioner in the } \\
\text { equipment room }\end{array}$ & 459.41 & 149.69 & 309.72 & $67.4 \%$ & $48.9 \%$ \\
\hline Air conditioner in the office & 356.04 & 131.13 & 224.91 & $63.2 \%$ & $35.5 \%$ \\
\hline Water dispenser & 18.46 & 10.78 & 7.68 & $41.6 \%$ & $1.2 \%$ \\
\hline Indoor lighting & 10.89 & 7.86 & 3.03 & $27.8 \%$ & $0.5 \%$ \\
\hline Outdoor lighting & 121.48 & 33.5 & 87.98 & $72.4 \%$ & $13.9 \%$ \\
\hline Total amount & 966.28 & 332.96 & 633.32 & $65.5 \%$ & $100 \%$ \\
\hline
\end{tabular}

As can be seen from Table 4, the most crucial energy-saving source of the pilot project is air conditioning. The energy-saving effect of air-conditioning is very significant, and can even reach $65.6 \%$, which is much higher than the energy-saving level in the optimization simulation. This is because the optimization strategy is implemented in winter, when the outdoor temperature is within the interval of $\left[20^{\circ} \mathrm{C}, 28^{\circ} \mathrm{C}\right]$, and before optimization the setting temperature was $22^{\circ} \mathrm{C}$. This is approximate to the outdoor temperature, so the operation of air conditioning is not necessary most of the time, resulting in a large amount of waste. After implementing the optimization strategy, most of the meaningless cooling operation power and invalid standby power was saved. Taking the three air conditioners in the central control room as an example, the operation before and after energy saving is shown in Figure 8.

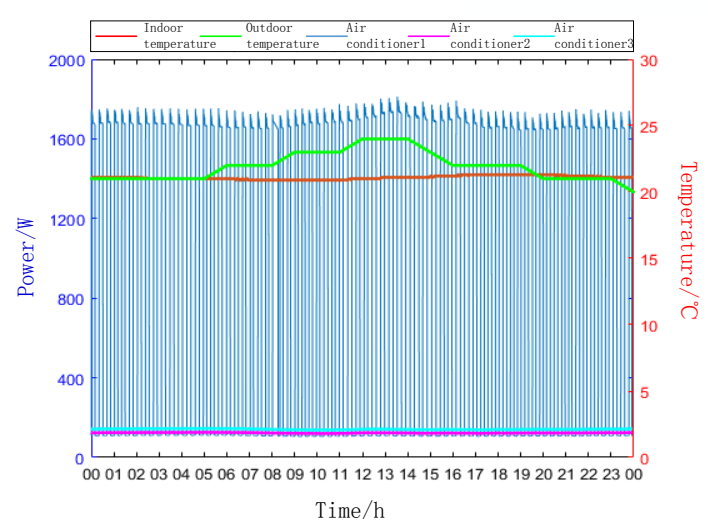

(a)

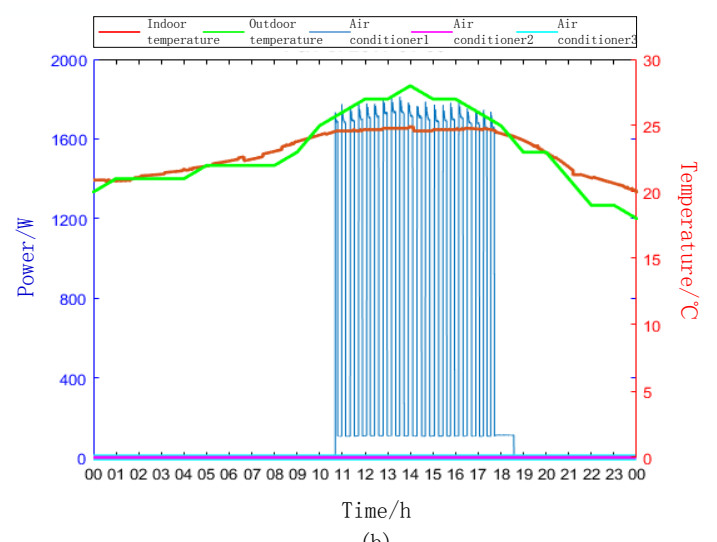

(b)

Figure 8. Energy saving optimization for air-conditioners in the central control room. (a) The operation of air conditioners and temperature before optimization. (b) The operation of air conditioners and temperature after optimization.

\section{Conclusions}

This paper proposes an optimal energy-saving strategy for HEMS, which considers bounded customer rationality. Three crucial contributions are made by this paper, including a general electric appliance utility function, a novel concept called the energy-saving cost incorporating prospect theory, and an energy-saving optimization model. The algorithm is first tested in a simulation system, and a case study is also set up based on real field tests from a pilot project in Guangdong province, China, in which air-conditioners, lighting, and some other popular electric appliances are included. The total energy-saving rate reaches $65.5 \%$ after the proposed energy-saving program is deployed. The benchmark test shows our optimal strategy is able to considerably save electric energy for residents, while ensuring customer comfort satisfaction. Limitations of this paper are as follows. First, the uncertainty of the environment is not considered. For example, temperature spikes can occur which may impact the results. Additionally, different electric retail rates are not considered in this 
paper, such as time of use and real time price. As a result, considering more practical environment conditions should be the focus of future research.

Author Contributions: Writing-original draft, G.L.; Writing-review \& editing, F.W. and S.F.; Data curation, Y.Y.; Methodology, F.P.; Software, S.Z.; Funding acquisition, G.L.; Project administration, G.L.; Supervision, S.F.

Funding: This research was funded by Science and Technology Project of China Southern Power Grid Co., Ltd. (GDKJXM20161607) and The National Natural Science Foundation of China (51877134).

Conflicts of Interest: The authors declare no conflict of interest.

\section{References}

1. Gottwalt, S.; Gärttner, J.; Schmeck, H.; Weinhardt, C. Modeling and valuation of residential demand flexibility for renewable energy integration. IEEE Trans. Smart Grid 2017, 8, 2565-2574. [CrossRef]

2. Jia, K.; Wang, Z.; Fan, S.; Zhai, S.; He, G. Data-Centric Approach: A Novel Systematic Approach for Cyber Physical System Heterogeneity in Smart Grid. IEEJ Trans. Electr. Electron. Eng. 2019. [CrossRef]

3. Pecorella, T.; Pierucci, L.; Nizzi, F. “Network Sentiment” Framework to Improve Security and Privacy for Smart Home. Future Internet 2018, 10, 125. [CrossRef]

4. He, J.; Xiao, Q.; He, P.; Pathan, M.S. An Adaptive Privacy Protection Method for Smart Home Environments Using Supervised Learning. Future Internet 2017, 9, 7. [CrossRef]

5. Fan, S.; Jia, K.; Guo, B.; Jiang, L.; Wang, Z.; He, G. Collaborative Optimal Operation Strategy for Decentralized Electric Heating Loads. Autom. Electr. Power Syst. 2017, 41, 20-29.

6. Alonso, S.; Morán, A.; Prada, M.Á.; Reguera, P.; Fuertes, J.J.; Domínguez, M. A Data-Driven Approach for Enhancing the Efficiency in Chiller Plants: A Hospital Case Study. Energies 2019, 12, 827. [CrossRef]

7. Gomes, L.; Ramos, C.; Jozi, A.; Serra, B.; Paiva, L.; Vale, Z. IoH: A Platform for the Intelligence of Home with a Context Awareness and Ambient Intelligence Approach. Future Internet 2019, 11, 58. [CrossRef]

8. Pan, F.; Lin, G.; Lin, J.; Fan, S.; He, G.; Jia, K. Design and Simulation of the Autonomous Decentralized Dispatching System of Generalized Demand Side Resources. In Proceedings of the 2018 IEEE Innovative Smart Grid Technologies-Asia (ISGT Asia), Singapore, 22-25 May 2018.

9. Jia, K.; Xiao, J.; Fan, S.; He, G. A MQTT/MQTT-SN-Based User Energy Management System for Automated Residential Demand Response: Formal Verification and Cyber-Physical Performance Evaluation. Appl. Sci. 2018, 8, 1035. [CrossRef]

10. Diaz Lozano Patino, E.; Siegel, J.A. Indoor environmental quality in social housing: A literature review. Build. Environ. 2018, 131, 231-241. [CrossRef]

11. Caniato, M.; Bettarello, F.; Ferluga, A.; Marsich, L.; Schmid, C.; Fausti, P. Thermal and acoustic performance expectations on timber buildings. Build. Acoust. 2017, 24, 219-237. [CrossRef]

12. Gupta, R.; Chandiwala, S. Understanding occupants: Feedback techniques for large-scale low-carbon domestic refurbishments. Build. Res. Inf. 2010, 38, 530-548. [CrossRef]

13. Fan, S.; He, G.; Jia, K.; Wang, Z. A Novel Distributed Large-Scale Demand Response Scheme in High Proportion Renewable Energy Sources Integration Power Systems. Appl. Sci. 2018, 8, 452. [CrossRef]

14. Fan, S.; He, G.; Guo, B.; Wang, Z. A user energy management system (UEMS)-based microgrid economic dispatch model. In Proceedings of the IEEE Asia-Pacific Power \& Energy Engineering Conference, Bangalore, India, 8-10 November 2018.

15. Mohajeryami, S.; Moghaddam, I.N.; Doostan, M.; Vatani, B.; Schwarz, P. A novel economic model for price-based demand response. Electr. Power Syst. Res. 2016, 135, 1-9. [CrossRef]

16. Lin, C.-M.; Wu, C.-Y.; Tseng, K.-Y.; Ku, C.-C.; Lin, S.-F. Applying Two-Stage Differential Evolution for Energy Saving in Optimal Chiller Loading. Energies 2019, 12, 622. [CrossRef]

17. Odkhuu, N.; Lee, K.-B.; A. Ahmed, M.; Kim, Y.-C. Optimal Energy Management of V2B with RES and ESS for Peak Load Minimization. Appl. Sci. 2018, 8, 2125. [CrossRef]

18. Tversky, A.; Kahneman, D. Advances in prospect theory: Cumulative representation of uncertainty. J. Risk Uncertain. 1992, 5, 297-323. [CrossRef] 
19. Jia, K.; He, G.; Yang, L.; Zhou, N. Preference Analyses of Residential Appliances in Demand Response: A Novel Perspective Based on Behavioral Economics. In Proceedings of the 2018 IEEE PES Innovative Smart Grid Technologies Conference Europe (ISGT-Europe), Sarajevo, Bosnia and Herzegovina, 21-25 October 2018.

20. Jia, K.; He, G.; Zhai, S.; Lin, G.; Lu, S.; Pan, F. Utility-Based Real-Time Estimation of Appliance Dispatching Cost for Residential Energy Management. In Proceedings of the 2018 IEEE Innovative Smart Grid Technologies-Asia (ISGT Asia), Singapore, 22-25 May 2018.

21. Wang, J.; Fang, K.; Dai, J.; Yang, Y.; Zhou, Y. Optimal Scheduling of Industrial Task-Continuous Load Management for Smart Power Utilization. Appl. Sci. 2017, 7, 281. [CrossRef]

22. Bradac, Z.; Kaczmarczyk, V.; Fiedler, P. Optimal Scheduling of Domestic Appliances via MILP. Energies 2015, 8, 217-232. [CrossRef]

23. Singh, M.; Jha, R.C. Object-Oriented Usability Indices for Multi-Objective Demand Side Management Using Teaching-Learning Based Optimization. Energies 2019, 12, 370. [CrossRef]

24. Dunkelberg, H.; Sondermann, M.; Meschede, H.; Hesselbach, J. Assessment of Flexibilisation Potential by Changing Energy Sources Using Monte Carlo Simulation. Energies 2019, 12, 711. [CrossRef]

25. Lin, G.; Pan, F.; Yang, Y.; Yang, L.; He, G.; Fan, S. The Pattern Recognition of Residential Power Consumption Based on HMM. In Proceedings of the 2018 IEEE Innovative Smart Grid Technologies-Asia (ISGT Asia), Singapore, 22-25 May 2018; pp. 413-418.

26. Liu, M.; Wu, Y.; Wu, R.; Fan, S.; He, G.; Jia, K. User-Side Self-Approximate Optimization Method and Its Application Based on Power Utility and Electrical Appliance Parameter Characterization. Electr. Power Constr. 2017. [CrossRef]

27. Kwac, J.; Flora, J.; Rajagopal, R. Household Energy Consumption Segmentation Using Hourly Data. IEEE Trans. Smart Grid 2014, 5, 420-430. [CrossRef]

(C) 2019 by the authors. Licensee MDPI, Basel, Switzerland. This article is an open access article distributed under the terms and conditions of the Creative Commons Attribution (CC BY) license (http:/ / creativecommons.org/licenses/by/4.0/). 